Introduction Successful PCI for CTO may confer an improved prognosis and a reduction in major adverse cardiac events (MACE). However most trials have included occlusions of short duration (less than 4 weeks). In this study we assessed the impact of PCI on LV function in patients with true CTOs (TIMI flow grade 0 and greater than 12 weeks duration) using serial CMR imaging as well as the predictive value of late gadolinium enhancement when performed prior to revascularisation.

Methods Thirty patients referred for PCI to a single vessel CTO underwent CMR examination prior to and 6 months after PCI. Technical success was defined as recanalisation of the occluded vessel and DES implantation with a final residual diameter stenosis $<30 \%$. LV function and infarct size were assessed using a 1.5T GE MRI system. Segmental wall thickening (SWT) was measured within the perfusion territory of the CTO using the 16-segment model and segments were dysfunctional if the SWT was $\leq 45 \%$. The transmural extent of infarction (TEI) was calculated by dividing the hyperenhanced area by the total area $\times 100$; a score of $\leq 25 \%$ were considered viable.

Results Technical success was achieved in 19 of the 30 patients $(63 \%)$. CTO duration was greater in patients with failed revascularisation but other baseline demographics were well matched between groups (Abstract 31 table 1). PCI-CTO success resulted in a significant increase in LVEF when compared to both baseline $(50 \pm 13$ vs $54 \pm 11 ; \mathrm{p}<0.01)$ and with PCI-CTO failure $(11.8 \pm 19.8$ vs $-2.3 \pm 5.1, \mathrm{p}<0.01$, Abstract 31 figure 1). In dysfunctional but viable segments only PCI success conferred a significant improvement in SWT compared to baseline ( $26 \pm 6$ vs $40 \pm 10$; $p<0.001$, Abstract 31 figure 2). There were no episodes of MACE in either group at 21 months follow-up.

Abstract 31 Table 1

\begin{tabular}{lllll}
\hline & Total $(\mathbf{n = 3 0})$ & $\begin{array}{l}\text { CTO-PCI Success } \\
(\mathbf{n = 1 9 )}\end{array}$ & $\begin{array}{l}\text { CTO-PCI Failure } \\
(\mathbf{n = 1 1})\end{array}$ & p value \\
\hline Age/ years & $62.2 \pm 10.2$ & $62.4 \pm 9.8$ & $61.8 \pm 11.4$ & 0.89 \\
Male, n (\%) & $25(83)$ & $14(74)$ & $11(100)$ & 0.13 \\
CCS Anginal Class & $2.13 \pm 0.68$ & $2.21 \pm 0.63$ & $2.0 \pm 0.77$ & 0.42 \\
LVEF/ \% & $53.0 \pm 11.6$ & $50.3 \pm 12.6$ & $57.6 \pm 8.1$ & 0.09 \\
CTO duration, months & $36.9 \pm 70.8$ & $12.6 \pm 26.4$ & $78.8 \pm 101.1$ & 0.01 \\
Vessel, n (\%) & & & & \\
$\quad$ RCA & $16(53)$ & $9(47)$ & $7(64)$ & 0.35 \\
$\quad 11(37)$ & $7(37)$ & $4(36)$ & \\
$\quad$ LAD & $3(10)$ & $3(16)$ & 0 & 0.61 \\
Prior MI, n (\%) & $17(59)$ & $11(58)$ & $6(56)$ & 0.61 \\
Diabetes Mellitus, n (\%) & $7(23)$ & $5(26)$ & $2(18)$ & 0.61 \\
Hypertension & $23(77)$ & $14(74)$ & $9(82)$ & \\
\hline
\end{tabular}

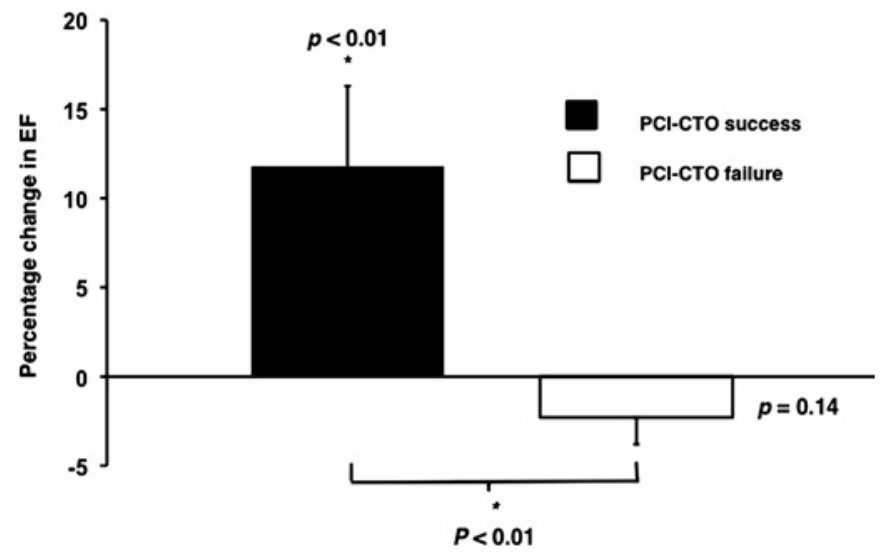

Abstract 31 Figure 1

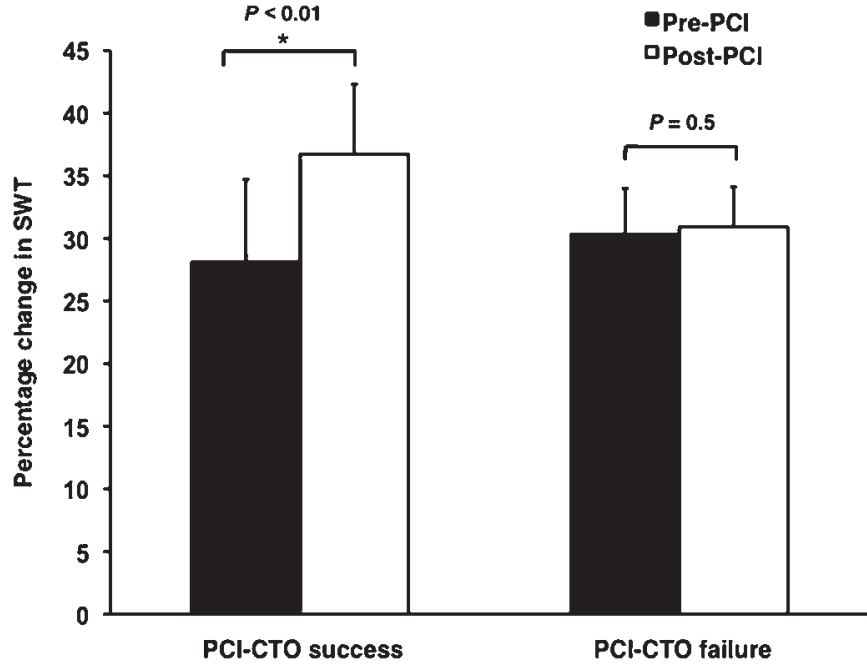

Abstract 31 Figure 2

Conclusion PCI-CTO success of true CTOs can improve global LV function. The TEI, assessed with CMR, can be used to help predict improvements in regional wall function. PCI-CTO failure was not associated with increased MACE at medium-term follow-up.

\section{DOES COMPLETE REVASCULARISATION CONFERS A LONG TERM SURVIVAL BENEFIT IN PATIENTS WITH CHRONICALLY OCCLUDED CORONARY VESSELS?}

doi:10.1136/heartjnl-2011-300198.32

N H Shah, M F Khan, T Ungvari, P H Loh, L Buchanan, A Hoye, R M Oliver, S Thackray, J L Caplin, M F Alamgir. Castle Hill Hospital, Kingston upon Hull, UK

Introduction Chronic total occlusion (CTO) of coronary vessels is a relatively common finding on diagnostic angiography. There has been increasing interest in this clinically important area with development of technologies resulting in improved recanalisation rates. However, long term survival data in this cohort is lacking. In this study we looked at survival of patients in whom complete, successful revascularisation was achieved.

Methods We identified consecutive patients, found to have CTO of at least one vessel of more than 1-month duration, on angiography performed between January 1999 and August 2000 in a single tertiary centre. We used a dedicated database to record data on variables and used central National Health Service database to obtain survival data. Results were analysed using SPSS statistics version 17.

Results We included 331 patients in the analysis. Mean age was 56.8 \pm 19.8 years, $76.1 \%$ were male and $21.8 \%(n=71)$ were diabetic. Mean duration of CTO was $29.5 \pm 25.9$ months and was only reliably estimated in $82.5 \%$ of cases. Median follow-up duration was $10.09 \pm 3.3$ years. Complete revascularisation was successfully achieved in $53.5 \%(n=177)$ patients, while $46.5 \%(n=154)$ were either treated medically from the outset or had failed or incomplete revascularisation. Both groups were age matched. Overall 10-year survival was $66.5 \%$; those with complete revascularisation had significantly improved survival over those with incomplete revascularisation or medical therapy $(75.1 \%$ vs $56.5 \%$, p $<0.001)$.

Conclusion Complete revascularisation confers a significant long term survival in patients with CTO and underscores the importance of improved recanalisation rates when performing angioplasty in this patient group. Overall survival was relatively poor and emphasises the importance of optimal medical therapy in this cohort. 


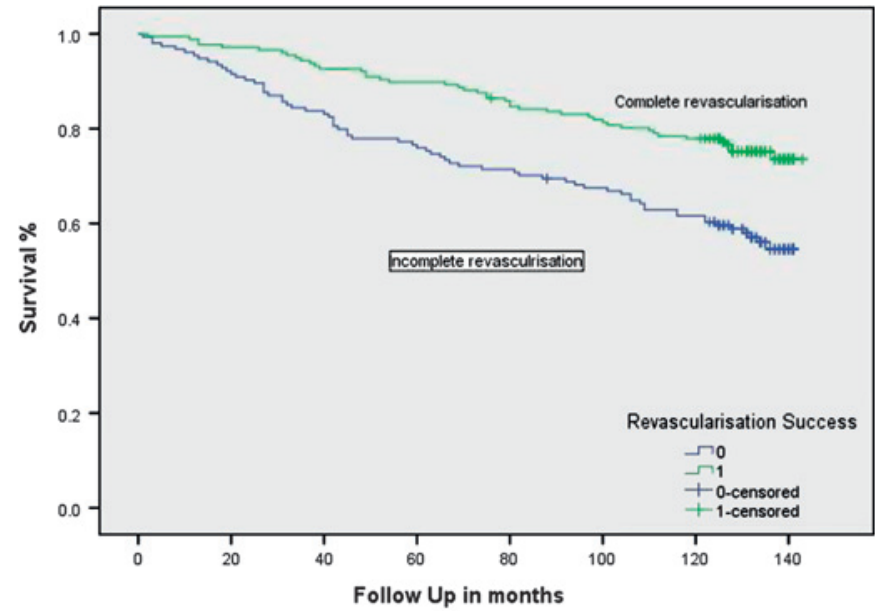

Revascularisation and Survival

Abstract 32 Figure 1

\begin{tabular}{l}
\hline 33 COMPLETENESS OF REVASCULARISATION PREDICTS \\
MORTALITY FOLLOWING PERCUTANEOUS CORONARY \\
INTERVENTION: UTILITY OF THE BCIS-1 JEOPARDY SCORE
\end{tabular}

doi:10.1136/heartjnl-2011-300198.33

K De Silva, G Morton, P Sicard, E Chong, A Indermeuhle, B Clapp, M Thomas, S Redwood, D Perera. St. Thomas' Hospital, King's College London, London, UK

Introduction Many coronary-scoring systems are complicated to use on a day-to-day basis, have varying degrees of reproducibility and exclude important subsets of patients such as those with previous coronary artery bypass grafts (CABG) or left main stem (LMS) disease (Abstract 33 table 1). The recently described BCIS-1 Myocardial Jeopardy score (BCIS-1 JS), a modification of the Duke Jeopardy score to include LMS and CABG, is simple to use and overcomes many of these limitations. We assessed the prognostic relevance of the BCIS-1 JS in patients undergoing percutaneous coronary intervention (PCI).

Abstract 33 Table 1

\begin{tabular}{llllll}
\hline & $\begin{array}{l}\text { Left Main } \\
\text { Stem Disease } \\
\text { classified }\end{array}$ & $\begin{array}{l}\text { Patients } \\
\text { with CABG } \\
\text { classified }\end{array}$ & $\begin{array}{l}\text { Ease } \\
\text { of use }\end{array}$ & $\begin{array}{l}\text { Relevance to } \\
\text { contemporary PCI }\end{array}$ & $\begin{array}{l}\text { Prognostic } \\
\text { validation }\end{array}$ \\
\hline $\begin{array}{l}\text { Duke Jeopardy } \\
\text { Score (Original) }\end{array}$ & $\mathrm{x}$ & $\mathrm{x}$ & $\sqrt{ }$ & $\mathrm{x}$ & $\sqrt{ }$ \\
Syntax Score & $\sqrt{ }$ & $\mathrm{x}$ & $\mathrm{x}$ & $\sqrt{ }$ & $\sqrt{ }$ \\
BCIS-1 JS & $\sqrt{ }$ & $\sqrt{ }$ & $\sqrt{ }$ & $\sqrt{ }$ & $\mathrm{x}$ \\
\hline
\end{tabular}

Methods Consecutive patients undergoing PCI between 2005 and 2009 a single cardiac centre were screened. Patients were eligible if they had undergone assessment of left ventricular function before PCI and the sample was enriched for coronary artery bypass graft (CABG) cases by using the following weighting - 1 CABG: 3 nonCABG. Clinicians (who were blinded to clinical or outcome data) scored diagnostic and procedural coronary angiograms. The BCIS-1 JS was recorded before and after PCI (range: 0 to 12) and a Revascularisation Index (RI) calculated as $\mathrm{RI}=\left(\mathrm{JS} \mathrm{S}_{\mathrm{PRE}}-\mathrm{JS}_{\mathrm{POST}}\right) / \mathrm{J} \mathrm{S}_{\mathrm{PRE}}$. $\mathrm{RI}=1.0$ indicates full revascularisation and 0 indicates no revascularisation. The primary end-point was all-cause mortality. Mortality data was captured by tracking the database of the UK Office of National statistics. Predictors of outcome were assessed by univariate and multivariate analyses.

Results 660 patients were included (68 19 years). $44 \%$ presented as acute coronary syndromes with $41 \%$ having left ventricular dysfunction. Over a follow-up period of $2.6 \pm 1.1$ years there were 42 deaths. All-cause mortality was inversely related to baseline BCIS-1 JS (HR 2.20 (1.34 to 3.62), $\mathrm{p}=0.002$ ) and to post-PCI BCIS-1 JS (HR 3.98 (2.33 to 6.78), $\mathrm{p}=0.0001$ ). Increasing degrees of revascularisation were associated with improved survival (Abstract 33 figure 1); a revascularisation index of $\geq 0.67$ was associated with a survival advantage compared to a $\mathrm{RI} \leq 0.66$ (HR 0.39 ( 0.24 to 0.54 ), $\mathrm{p}=0.0001$ ) (Abstract 33 table 2). A multiple regression model, incorporating age, acuity of presentation, LV function and renal failure, demonstrated that $\mathrm{RI}=0.67-1$ continued to be an independent predictor of survival (HR $0.5195 \%$ CI 0.35 to 0.81, p=0.004) (Abstract 33 figure 1).

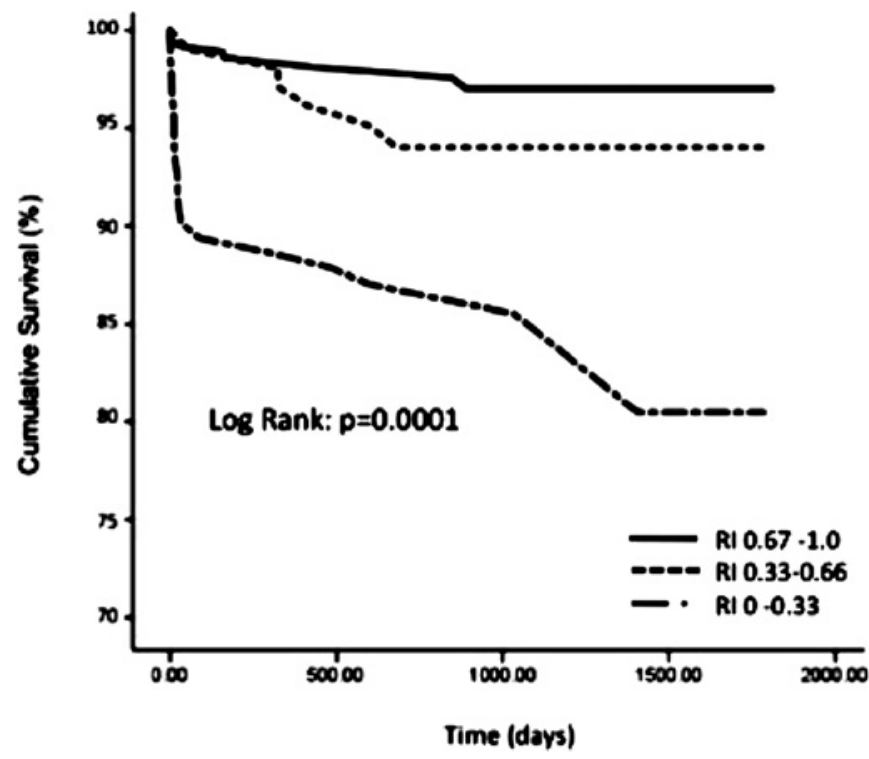

Abstract 33 Figure 1 Cumulative survival according to Revascularisation Index (RI).

Abstract 33 Table 2

\begin{tabular}{|c|c|c|c|c|}
\hline Variables & $\begin{array}{l}\text { Univariate analysis } \\
\text { HR }(95 \% \mathrm{CI})\end{array}$ & p value & $\begin{array}{l}\text { Multivariate } \\
\text { analysis HR }(95 \% \mathrm{CI})\end{array}$ & p value \\
\hline $\begin{array}{l}\text { Revascularisation } \\
\text { Index }(0.67-1)\end{array}$ & $0.36(0.24$ to 0.54$)$ & 0.0001 & $0.51(0.33$ to 0.81$)$ & 0.004 \\
\hline BCIS-1 JS pre PCI & 1.26 (1.14 to 1.39$)$ & 0.0001 & $1.14(0.65$ to 2.02$)$ & 0.65 \\
\hline BCIS-1 JS post PCI & 1.35 (1.23 to 1.48$)$ & 0.0001 & 1.78 (0.93 to 3.39$)$ & 0.08 \\
\hline LV impairment & 3.76 (2.53 to 5.58 ) & 0.0001 & $1.97(1.21$ to 3.20$)$ & 0.007 \\
\hline Age & 1.04 (1.01 to 1.08$)$ & 0.01 & $1.04(1.00$ to 1.08$)$ & 0.05 \\
\hline Renal dysfunction & $5.82(2.77$ to 12.24$)$ & 0.0001 & $3.74(1.60$ to 7.37$)$ & 0.002 \\
\hline $\begin{array}{l}\text { Acute coronary } \\
\text { syndrome }\end{array}$ & $2.31(1.24$ to 4.30$)$ & 0.008 & $1.30(0.63$ to 2.66$)$ & 0.47 \\
\hline Cardiogenic shock & 14.56 (6.45 to 32.88 ) & 0.0001 & $2.83(0.69$ to 11.54$)$ & 0.15 \\
\hline Previous CABG & $3.35(1.80$ to 6.25$)$ & 0.0001 & $1.83(0.88$ to 3.82$)$ & 0.10 \\
\hline
\end{tabular}

Conclusion The BCIS-1 Jeopardy Score predicts mortality following PCI. Furthermore, it can be used to assess the degree of revascularisation, with more complete revascularisation (RI $\geq 0.67)$ conferring a survival advantage in the medium term.

\section{COMPARISON OF PCI VS CABG IN INSULIN TREATED AND NON-INSULIN TREATED DIABETIC PATIENTS IN THE CARDIA TRIAL}

doi:10.1136/heartjnl-2011-300198.34

${ }^{1} \mathrm{~A}$ Baumbach, ${ }^{2} \mathrm{~S}$ Kesavan, ${ }^{3} \mathrm{~K}$ Beatt, ${ }^{4} \mathrm{E}$ Cruddas, ${ }^{4} \mathrm{M}$ Flather, ${ }^{2} \mathrm{G}$ Angelini, ${ }^{5} \mathrm{R}$ Hall, ${ }^{6}$ A Kapur. ${ }^{1}$ Bristol Heart Institute, Bristol, UK; ${ }^{2}$ Bristol Heart Institute, Bristol, UK; 